\title{
The early type contact binary system V 382 Cygni
}

\author{
Ö.L. Değirmenci ${ }^{1}$, C. Sezer ${ }^{1}$, O. Demircan ${ }^{2}$, A. Erdem ${ }^{3}$, S. Özdemir ${ }^{2}$, H. Ak ${ }^{2}$, and B. Albayrak ${ }^{2}$ \\ 1 Ege University Observatory, Science Faculty, Izmir, Turkey \\ 2 Ankara University Observatory, Science Faculty, Ankara, Turkey \\ 3 Department of Physics, Faculty of Arts and Sciences, Çanakkale Onsekiz Mart University, Çanakkale, Turkey
}

Received March 27; accepted May 26, 1998

\begin{abstract}
New photometric data set of the early type contact binary V 382 Cyg have been analyzed with emphasis on determining the photometric mass ratio of the system. The result $(q=0.68)$ supports the value given by Popper \& Hill (1991). A study of the system using new data shows a period increase of about $3.28 \mathrm{~s}$ per century, which corresponds to a very large rate $\sim 5.010^{-6} M_{\odot} / \mathrm{yr}$ of mass transfer from the less massive to the more massive component under the conservative mass transfer hypothesis.
\end{abstract}

Key words: stars: binaries: eclipsing — stars: individual: V 382 Cyg

\section{Introduction}

The variability of V 382 Cygni (= HD $228854=\mathrm{BD}+$ $35^{\circ} 4062$ ) was discovered by Morgenroth (1935). The eclipsing character of the system was suggested photographically by Petrov (1946). Petrov noted that the light curve is $\beta$ Lyrae type and the depths of the minima are nearly equal. The photographic light curve of Petrov was analyzed by Albo (1958). Pearce (1952) published the spectroscopic elements of the system and considered the spectral types of the components as O6.5 and O 7.5. He found that the orbital velocities are exceedingly large $\left(K 1+K 2=710 \mathrm{~km} \mathrm{~s}^{-1}\right)$. He also derived the masses of the components as $37.3 M_{\odot}$ and $32.7 M_{\odot}$ and the length of the semi-major axis of the relative orbit $(a \sin i)$ as $26.4 R_{\odot}$. Photoelectric $U B V$ light curves of the system were obtained by Landolt (1964, 1975). Landolt's (1964) light curves were analyzed by himself using the Russell-Merrill Method. Landolt's (1975) light curves were analyzed using Wood's WINK method by Cester et al. (1978) and using the Wilson-Devinney method by Devinney \& Twigg (1974). According to Cester

Send offprint requests to: Ö.L. Değirmenci et al., the system is not a detached but a contact binary. They gave the masses of the components as $38 M_{\odot}$ and $33.3 M_{\odot}(q=0.88)$. Koch et al. (1979) have obtained the IUE spectrum of V 382 Cygni. The period variation of the system was studied by Mayer (1980, 1987), and Mayer et al. (1991). Popper \& Hill (1991) analyzed the spectroscopic observations of Popper (1978) and obtained orbital solutions. They also obtained the absolute elements of the system. Popper \& Hill gave the masses of the components as $32.6 M_{\odot}$ and $22.9 M_{\odot}(q=0.70)$. Popper $(1980)$ lists the spectral types for $\mathrm{V} 382 \mathrm{Cyg}$ as $\mathrm{O} 7.3 \mathrm{~V}$ and $\mathrm{O} 7.7 \mathrm{~V}$.

A recent spectroscopic investigation of the system was published by Harries et al. (1997). They also solved Landolt's (1975) light curves using the LIGHT 2 method. They found that V 382 Cygni is a contact system with masses of the components as $26 M_{\odot}$ and $19.3 M_{\odot}(q=$ $0.74)$. These values of the masses are significantly lower than the previous ones. We decided to observe the system to obtain complete photoelectric light curves and reliable photometric solutions of V 382 Cygni.

\section{Observations}

The system was observed photoelectrically at the Ege University Observatory (EUO) on 16 nights and at the Ankara University Observatory (AUO) on 13 nights during the summer of 1995. The observations were obtained with the 48-cm Cassegrain reflector of EUO and the 30-cm Maksutov telescope of AUO. The SSP5A photometer was used on both telescopes. A total of 871 blue and 853 yellow observational points have been obtained at EUO. BD $+37^{\circ} 3879$ and $\mathrm{BD}+36^{\circ} 4095$ were used as comparison and check stars. A total of 597 observational points in each color of ultraviolet, blue and yellow have been obtained at AUO, and BD $+36^{\circ} 4095$ was used as comparison star. No variations in brightness were detected for the comparison stars. The observations of comparison stars were used to determine the nightly extinction coefficients in each color separately. All the instrumental differential 


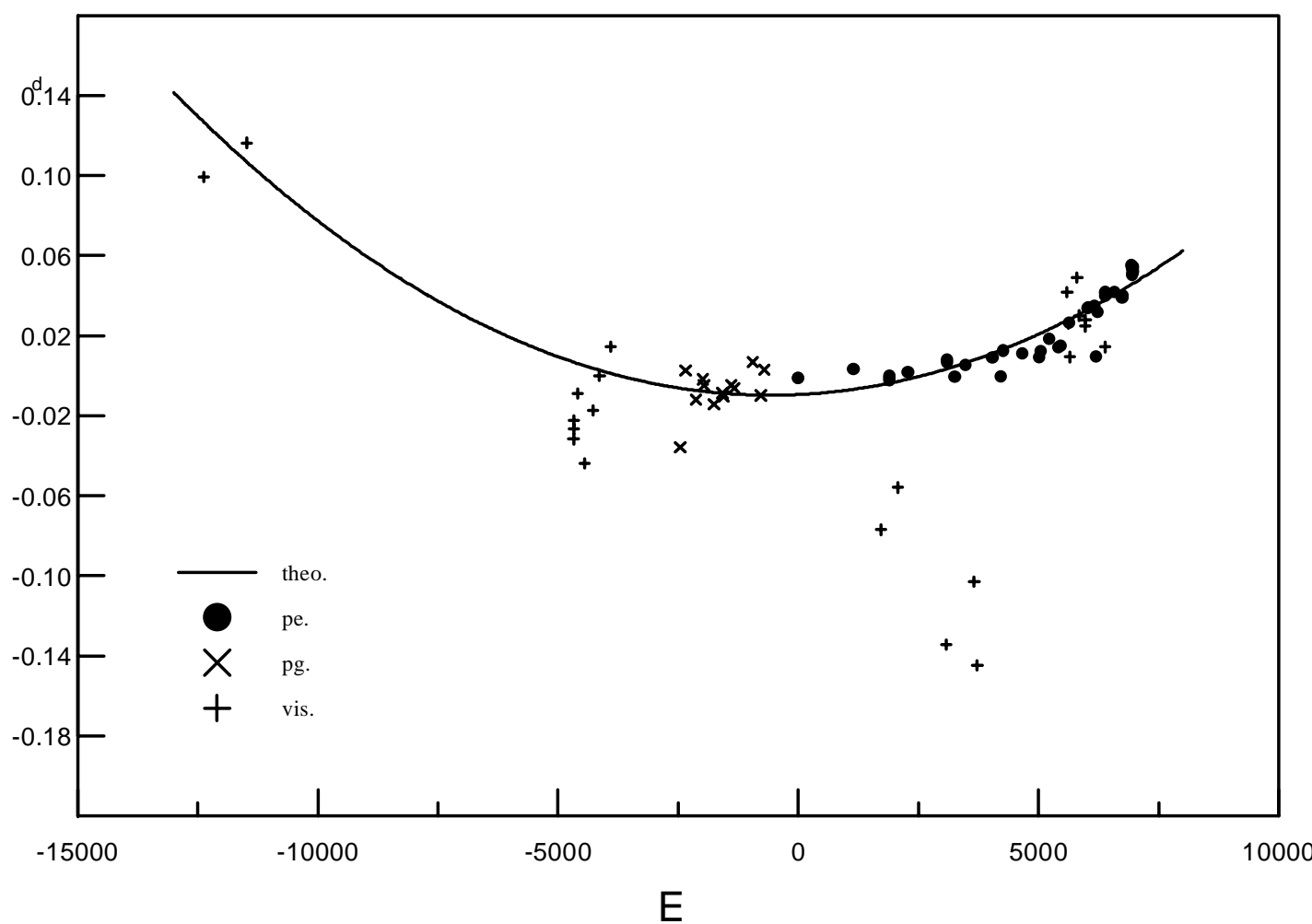

Fig. 1. The O-C diagram for V 382 Cygni

magnitudes (in the sense variable minus comparison) were corrected for the atmospheric extinction. The differential observations for which the probable error of a single observation was estimated to be \pm 0 . 007 at EUO and \pm 0 . 01 at AUO for all wavelengths are available on request from the authors.

\section{Period study}

During the observations, five primary and four secondary times of minima were obtained and given in Table 1. All available times of minima were collected from the literature. Together with the new times of minima from Table 1 , they were used in forming the $(\mathrm{O}-\mathrm{C})$ diagram (see Fig. 1). The light elements given by Landolt (1975) were used in estimating $C$ values. The $(\mathrm{O}-\mathrm{C})$ diagram in Fig. 1 shows a parabolic variation indicating a continuous increase in period. The period increase is found to be $3.28 \pm 0.26$ seconds per century. The corresponding quadratic light elements were obtained to be

Hel Min I JD $=2436814.7642+1$. $8855132 E+0.9810^{-9} E^{2}$.

$$
\pm 18 \quad \pm 5 \quad \pm 8
$$

If the period increase is due to conservative mass transfer, then the mass transfer rate from the less massive to the more massive component should be about $5.010^{-6}$ solar mass per year. The phases of the light variation of
Table 1. New times of minima of V 382 Cygni

\begin{tabular}{lll}
\hline Hel JD 2400000+ & Min. & Observer \\
\hline 49880.4846 & II & AUO \\
49913.4766 & I & AUO \\
49915.3654 & I & AUO \\
49929.5074 & II & AUO \\
49930.4476 & I & EUO \\
49931.3909 & II & EUO \\
49945.5342 & I & EUO \\
49946.4757 & II & EUO \\
49947.4171 & I & EUO \\
\hline
\end{tabular}

the system were calculated with the following linear light elements:

Hel Min I JD = $2436814.6751+1.88553364 E$,

which were obtained from the recent photoelectric times of minima.

\section{Photometric solutions}

The Wilson \& Devinney (1971) method was applied in solving the present light curves of V 382 Cygni. This method has been described by many authors. For our solutions, the revised version of the program (Wilson 1992) was used. The method assumes the star surfaces to be 


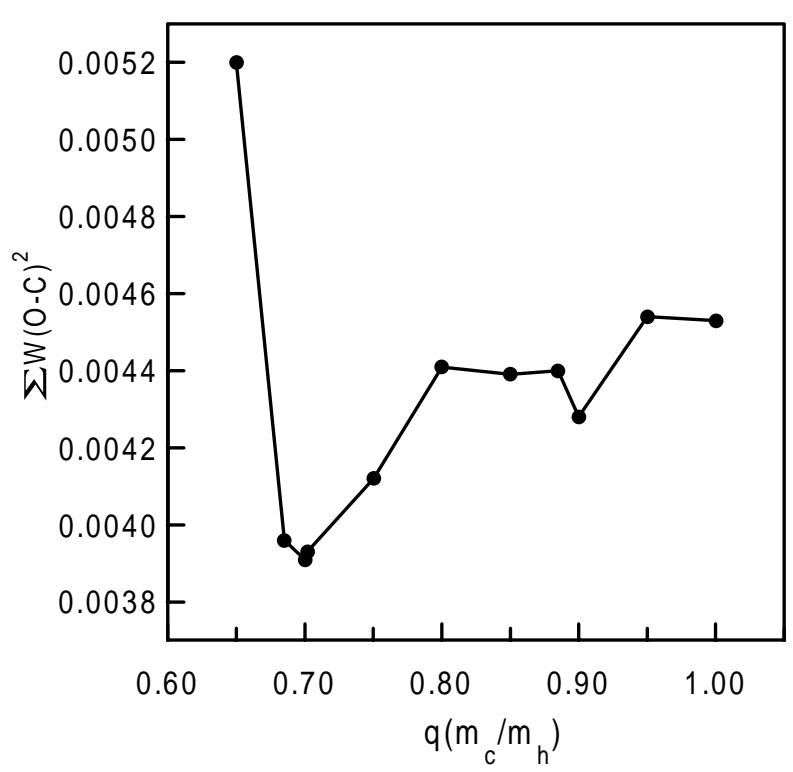

Fig. 2. The behavior $\Sigma W(\mathrm{O}-\mathrm{C})^{2}$ as a function of the mass ratio $q$

equipotentials and computes the light curves as a function of the following parameters: the orbital inclination $i$, surface potentials $\Omega_{\mathrm{h}, \mathrm{c}}$, flux-weighted average surface temperatures $T_{\mathrm{h}, \mathrm{c}}$, mass ratio $q=m_{\mathrm{c}} / m_{\mathrm{h}}$, unnormalized monochromatic luminosities $L_{\mathrm{h}, \mathrm{c}}$, linear limb-darkening coefficients $x_{\mathrm{h}, \mathrm{c}}$, gravity darkening exponents $g_{\mathrm{h}, \mathrm{c}}$, and bolometric albedos $A_{\mathrm{h}, \mathrm{c}}$. Throughout this paper, the subscripts $h$ and $c$ refer to the primary (hotter) and secondary (cooler) component, respectively. For the solution of the present light curves, first the independent sets of $B$ and $V$ observations made at two observatories were combined after normalization to form single $B$ and $V$ light curves. The observational points in each $B$ and $V$ light curves were then combined into normal points and weighted directly according to the number of individual observations including in a point. The temperature of the primary component was taken from Morton \& Adams (1968) as equal to $36100 \mathrm{~K}$, corresponding to the $\mathrm{O} 6.5$ spectral class given by Pearce (1952). This temperature is in accord with the discussion in Hilditch et al. (1996) and in Harries et al. (1997). The linear limb darkening coefficients were taken from Wade \& Rucinski (1985), the gravity darkening exponents and the bolometric albedos were set to be equal to 1.0 for radiative atmospheres. These parameters were kept constant during the iterations. The spectroscopic mass ratio of the system was given by Pearce (1952) as $q=m_{\mathrm{c}} / m_{\mathrm{h}}=0.88$. Popper \& Hill (1991) and Harries et al. (1997) obtained slightly different values for the mass ratio as 0.70 and 0.74 , respectively. Therefore, we decided to apply a $q$-search procedure for determining the photometric mass ratio of the system. For this, the $B$ and $V$ light curves were solved simultaneously by choosing $i, T_{\mathrm{c}}, \Omega_{\mathrm{h}, \mathrm{c}}\left(\Omega_{\mathrm{h}}=\Omega_{\mathrm{c}}\right), L_{\mathrm{h}}$ as adjustable param-

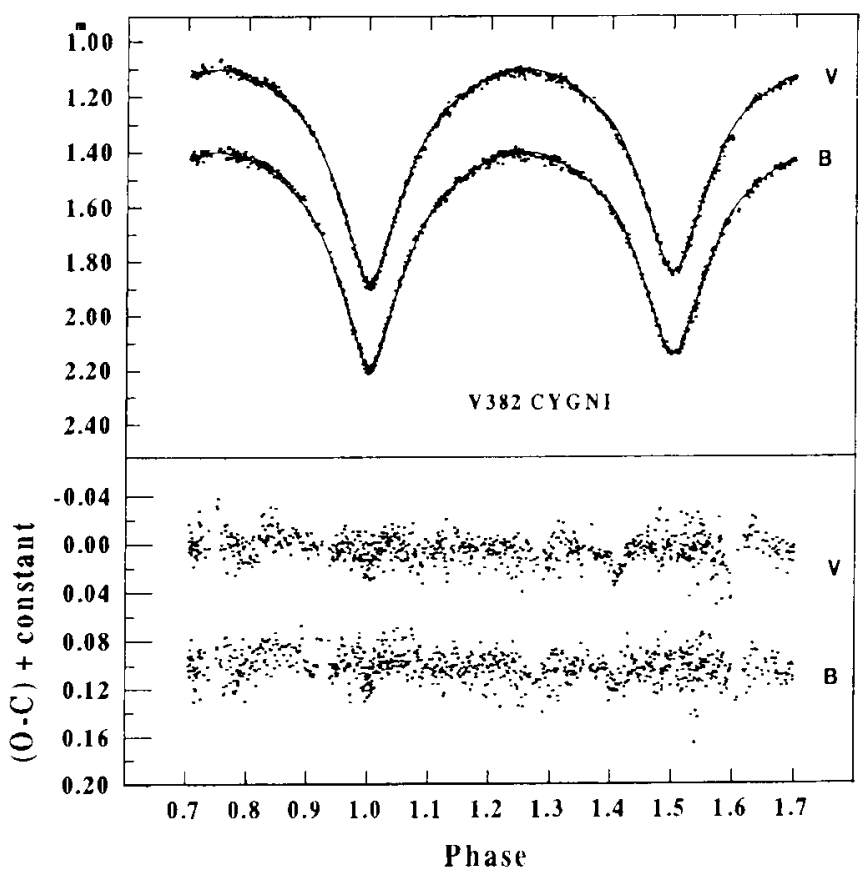

Fig. 3. EUO light curves of V 382 Cygni. The upper panel shows the theoretical $B$ and $V$ light curves (solid lines) formed by Wilson-Devinney model among the observations obtained at EUO, while the bottom panel shows the $(\mathrm{O}-\mathrm{C})$ differences between the observations and theoretical fits

eters. The analysis was made with contact configuration (i.e. MODE 3). The weighted sum of the squared residuals $\left[\sum W(\mathrm{O}-\mathrm{C})^{2}\right]$ for the corresponding mass ratios are shown in Fig. 2. As can be seen from the figure, the lowest value of $\left[\sum W(\mathrm{O}-\mathrm{C})^{2}\right]$ around $q=0.68$ supports the spectroscopic mass ratio $(q=0.702)$ given by Popper \& Hill (1991), which was used subsequently as a starting input parameter in the solutions. The convergent simultaneous solutions of the $B$ and $V$ light curves were obtained with the free parameters by iterating until the corrections on the parameters became smaller than the corresponding probable errors. Because of the larger scatter in the $U$ observations, the $U$ light curve has not been included in the analysis. The results of the present analysis are given in Table 2. The theoretical light curves calculated with the final elements obtained from simultaneous solution of the combined $B$ and $V$ light curves are shown in Fig. 3 and 4 among four observational light curves (two in $B$, and two in $V$ ) from two observatories. As seen from the figures, the agreements between theoretical and observational light curves are very good. The over contact configuration of V 382 Cygni calculated with the Roche model is shown in Fig. 5. The degree of overcontact is $22 \%$.

The absolute elements of the system were also obtained by combining our photometric results and the spectroscopic elements given by Harries et al. (1997). The results are given in Table 3. 
Table 2. The results obtained by the method of Wilson-Devinney

\begin{tabular}{llll}
\hline Parameter & $B$ & $V$ & $B \& V$ \\
\hline$i$ & $85.6 \pm 0.1$ & $85.7 \pm 0.1$ & $85.7 \pm 0.1$ \\
$x_{\mathrm{h}}$ & 0.24 & 0.20 & $0.24(\mathrm{~B}), 0.20(\mathrm{~V})$ \\
$x_{\mathrm{c}}$ & 0.24 & 0.20 & $0.24(\mathrm{~B}), 0.20 \mathrm{~V})$ \\
$A_{\mathrm{h}}$ & 1.0 & 1.0 & $1.0(\mathrm{~B}), 1.0(\mathrm{~V})$ \\
$A_{\mathrm{c}}$ & 1.0 & 1.0 & $1.0(\mathrm{~B}), 1.0(\mathrm{~V})$ \\
$g_{\mathrm{h}}$ & 1.0 & 1.0 & $1.0(\mathrm{~B}), 1.0(\mathrm{~V})$ \\
$g_{\mathrm{c}}$ & 1.0 & 1.0 & $1.0(\mathrm{~B}), 1.0(\mathrm{~V})$ \\
$T_{\mathrm{h}}$ & $36100 \mathrm{~K}$ & $36100 \mathrm{~K}$ & $36100 \mathrm{~K}$ \\
$T_{\mathrm{c}}$ & $34894 \pm 68 \mathrm{~K}$ & $34690 \pm 81 \mathrm{~K}$ & $34758 \pm 52 \mathrm{~K}$ \\
$\Omega_{\mathrm{h}}=\Omega_{\mathrm{c}}$ & $3.1354 \pm 0.0087$ & $3.1055 \pm 0.0077$ & $3.1184 \pm 0.0036$ \\
$q$ & $0.684 \pm 0.006$ & $0.675 \pm 0.005$ & $0.677 \pm 0.002$ \\
$L_{\mathrm{h}} /\left(L_{\mathrm{h}}+L_{\mathrm{c}}\right)$ & $0.5961 \pm 0.0020$ & $0.6001 \pm 0.0016$ & $0.6003 \pm 0.0009(\mathrm{~B})$ \\
& & & $0.5991 \pm 0.0009(\mathrm{~V})$ \\
$L_{\mathrm{c}} /\left(L_{\mathrm{h}}+L_{\mathrm{c}}\right)$ & 0.4039 & 0.3999 & $0.3997(\mathrm{~B}), 0.4009(\mathrm{~V})$ \\
$r_{\mathrm{h}}($ pole $)$ & 0.3999 & 0.4033 & 0.4016 \\
$r_{\mathrm{h}}($ side $)$ & 0.4248 & 0.4290 & 0.4269 \\
$r_{\mathrm{h}}($ back $)$ & 0.4599 & 0.4652 & 0.4624 \\
$r_{\mathrm{c}}($ pole $)$ & 0.3369 & 0.3382 & 0.3371 \\
$r_{\mathrm{c}}($ side $)$ & 0.3545 & 0.3562 & 0.3548 \\
$r_{\mathrm{c}}($ back $)$ & 0.3941 & 0.3975 & 0.3952 \\
{$\left[\sum W(\mathrm{O}-\mathrm{C})^{2}\right]$} & 0.0018 & 0.0021 & 0.0039 \\
\hline
\end{tabular}

Table 3. The absolute elements of V 382 Cygni

\begin{tabular}{|c|c|c|}
\hline & Primary & Secondary \\
\hline$M / M_{\odot}$ & $26.6 \pm 0.2$ & $18.0 \pm 0.2$ \\
\hline$R / R_{\odot}$ & $9.85 \pm 0.04$ & $8.30 \pm 0.03$ \\
\hline$M_{\mathrm{bol}}$ & $-8.1 \pm \quad 0.1$ & $-7^{\mathrm{m}} 6 \pm \quad 0.1$ \\
\hline $\log g(\operatorname{cgs})$ & $3.88 \pm 0.02$ & $3.86 \pm 0.02$ \\
\hline $\log L / L_{\odot}$ & $5.13 \pm 0.02$ & $4.93 \pm 0.02$ \\
\hline
\end{tabular}

\section{Results and discussion}

The Present data of V 382 Cyg is representable only by an overcontact model with fill-out parameter of $22 \%$. Most probably, the overcontactness of the system was formed by;

i) orbital shrinkage due to Roche Lobe overflow from the original more massive component and

ii) spin-orbit coupled angular momentum loss from the system, in addition to evolutionary expansion of the component stars, although radiative pressure works against the formation of contact configuration.

The results of our light curve solution are not much different from those given by Harries et al. (1997). They analyzed Landolt's photometric observations. The absolute dimensions in Table 3, which were obtained by combining our light curve solution with the radial velocity solution of Harries et al., are also not much different from those given by Harries et al. However, they are (particularly for the secondary component) 10-30 percent lower than earlier results (see e.g. Pearce 1952; Cester et al. 1978, and Popper \& Hill 1991).

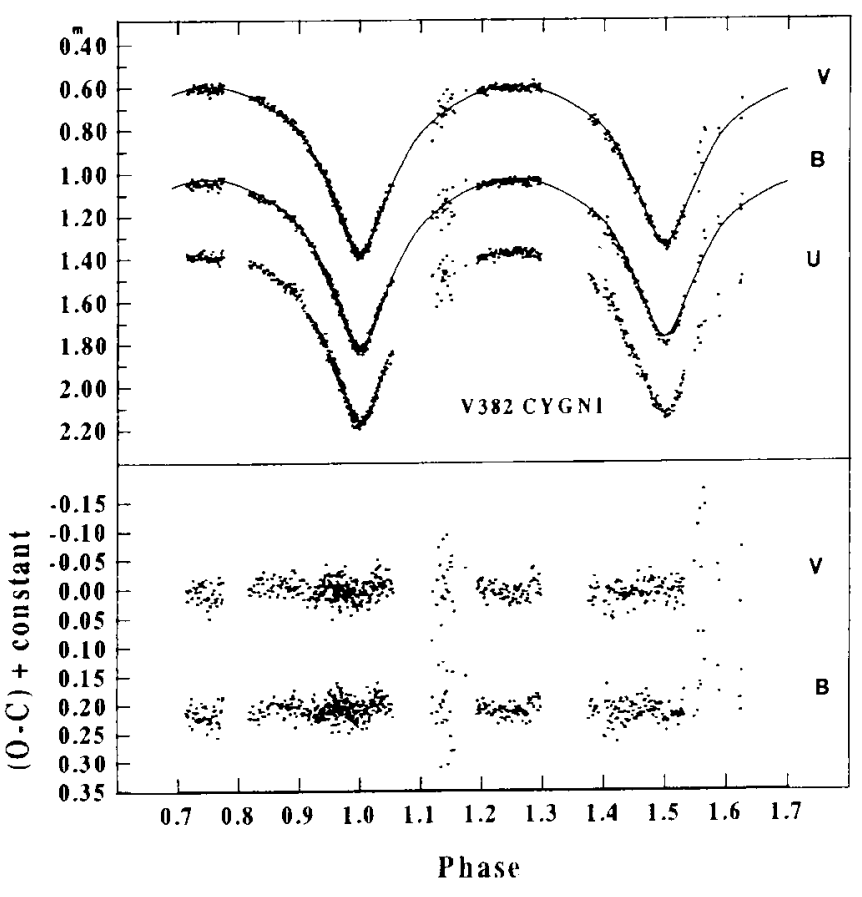

Fig. 4. AUO light curves of V 382 Cygni. The upper panel shows the theoretical $B$ and $V$ light curves (solid lines) formed by Wilson-Devinney model among the observations obtained at AUO, while the bottom panel shows the $(\mathrm{O}-\mathrm{C})$ differences between the observations and theoretical fits

The mass ratio $q$ is known to be a key parameter in the light curve solutions of eclipsing binaries. To determine the photometric mass ratio of the system, we applied a $q$-search procedure on our new data. Our result $(q=0.68)$ verifies the spectroscopic mass ratios given by 


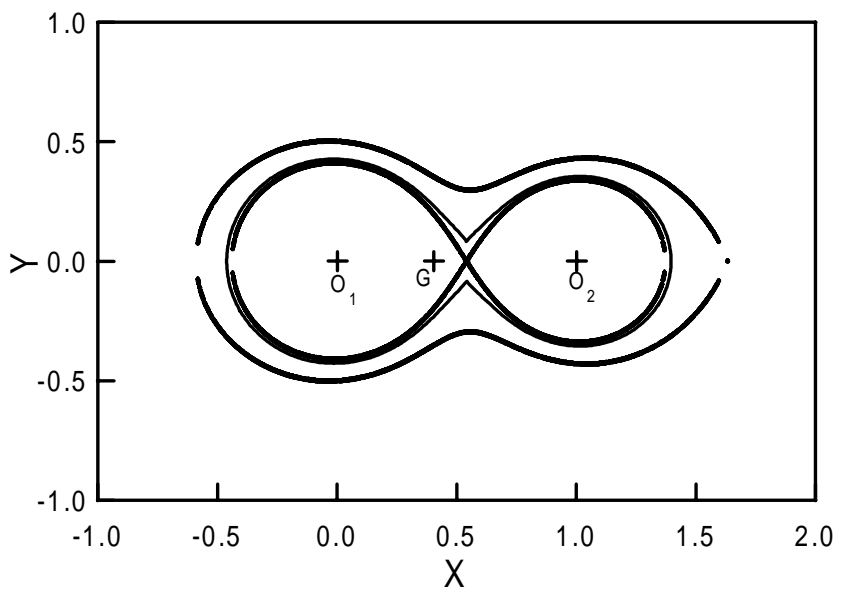

Fig. 5. The Roche configuration of V 382 Cygni for $q=0.677$

Popper \& Hill (1991) and Harries et al. (1997). According to Leung (1988), there is very good agreement, in general, between the photometric mass ratios and the spectroscopic mass ratios derived from cross-correlation techniques. Our result confirms this conclusion.

A period study using the new data together with published times of eclipse minima revealed an increase of about $3.28 \mathrm{~s}$ per century in the orbital period of the system. The secular period increase of contact binary systems is possible only by mass transfer from the less massive to the more massive component. By using the conservative mass transfer hypothesis, the mass transfer rate was found to be about $5.010^{-6} M_{\odot} /$ yr. This rate should be the minimum value, because any mass and momentum loss from such a spin-orbit coupled contact binary system tends to decrease the orbital period. Considerable mass loss due to a strong interacting wind from V 382 Cyg is inevitable. The light curve variability occuring on the rising branches of the eclipses and in the depths of the secondary minima (see Landolt 1975) and the P Cygni profiles of certain $U V$ lines (see Koch et al. 1979) are all indicative of mass loss from the system. Koch et al. (1979) estimated a mass loss rate of $410^{-5} M_{\odot} / \mathrm{yr}$, although rates of $510^{-7} M_{\odot} /$ yr are expected for $\mathrm{O} 6.5$ main-sequence stars (cf. Howarth \& Prinja 1989).

In summary, the secular period increase of the system requires a very large rate, at least $5.010^{-6} M_{\odot} / \mathrm{yr}$, of mass transfer from the less massive to the more massive component. The mass ratio of the system is already reversed. The orbital period of the system initially decreased very rapidly and the contact configuration should have been formed before the mass ratio reversal. The period should have been increasing since mass ratio reversal of the system. The mass loss from the system should decrease the rate of period increase of the system. The short term variations in the $(\mathrm{O}-\mathrm{C})$ diagram should be caused by irregularities in the mass transfer and mass loss.

Acknowledgements. This work was partly supported by the Scientific and Technical Research Council of Turkey under TBAG-AY/78.

\section{References}

Albo H., 1958, Variable Stars Bull. 12, 240

Cester B., Fedel B., Giuricin G., Mardirossian F., Mezzetti M., 1978, A\&AS 33, 91

Devinney E.J., Twigg L.W., 1974, BAAS 6, 335

Harries T.J., Hilditch R.W., Hill G., 1997, MNRAS 285, 277

Hilditch R.W., Harries T.J., Bell S.A., 1996, A\&A 314, 165

Howarth I.D., Prinja R.K., 1989, ApJS 69, 527

Koch R.H., Siah M.J., Fanelli M.N., 1979, PASP 91, 47

Landolt A.U., 1964, ApJ 140, 1494

Landolt A.U., 1975, PASP 87, 409

Leung K.C., 1988, in: Critical Observations versus Physical Models for Close Binary Systems, Leung K.C. (ed.), Gordon \& Breach Science Publishers, New York, p. 93

Mayer P., 1980, Bull. Astron. Inst. Czech. 31, 292

Mayer P., 1987, Bull. Astron. Inst. Czech. 38, 58

Mayer P., Wolf M., Tremko J., Niarchos P.G., 1991, Bull. Astron. Inst. Czech. 42, 225

Morgenroth O., 1935, Astron. Nachr. 255, 425

Morton D.C., Adams T.F., 1968, ApJ 151, 611

Pearce J.A., 1952, PASP 64, 219

Petrov A.A., 1946, Variable Stars Bull. 6, 72

Popper D.M., 1978, ApJL 220, L11

Popper D.M., 1980, ARA\&A 18, 115

Popper D.M., Hill G., 1991, AJ 101, 600

Wade R.A., Rucinski S.M., 1985, A\&AS 60, 471

Wilson R.E., 1992 (private communication)

Wilson R.E., Devinney E.J., 1971, ApJ 166, 605 